\title{
Structure and regulation of the gene for dGTP triphosphohydrolase from Escherichia coli
}

\author{
(dgt/optA/bacteriophage T7 gene 1.2 protein/promoter)
}

\author{
Susannah M. Wurgler and Charles C. Richardson \\ Department of Biological Chemistry and Molecular Pharmacology, Harvard Medical School, Boston, MA 02115 \\ Contributed by Charles C. Richardson, January 18, 1990
}

\begin{abstract}
Escherichia coli encodes an enzyme, deoxyguanosine triphosphate triphosphohydrolase (dGTPase, EC 3.1.5.1), that cleaves dGTP into deoxyguanosine and tripolyphosphate. An $E$. coli mutant, optA1, contains a 50-fold increased level of dGTPase and cannot support the growth of phage T7 defective gene 1.2, whose product is an inhibitor of dGTPase. The optA1 mutation maps to $3.6 \mathrm{~min}$ on the $E$. coli chromosome and is closely linked to dapD. We have isolated the gene encoding dGTPase (dgt) from wild-type $E$. coli and determined its nucleotide sequence. The dgt gene lies immediately upstream of $h t r A$ and 6 kilobases from dapD, in the same region as the optAl mutation. The dgt structural gene is 1515 base pairs, encoding a protein of 59,315 daltons, in agreement with the size and $\mathrm{N}$-terminal amino acid sequence of the purified protein. An $E$. coli strain containing a null allele has no detectable phenotype when grown at $30-42^{\circ} \mathrm{C}$ in rich medium. A transition of $\mathrm{C}$ to $\mathrm{T}$ in a potential promoter of $d g t$ is required for expression of the optAl phenotype.
\end{abstract}

Extracts of Escherichia coli contain a dGTPase (EC 3.1.5.1) that catalyzes the hydrolysis of dGTP to deoxyguanosine and tripolyphosphate $(1,2)$. E . coli optAl cells contain a 50 -fold higher level of dGTPase than do $E$. coli opt $A^{+}$cells as determined by activity $(2,3)$, protein yield during purification (3), and Western blots (4). Biochemical analysis of the optAI mutant has shown that the intracellular dGTP pool is 5-fold lower than that in wild-type cells (5), presumably due to the increased level of dGTPase. In addition, the replication of some bacteriophages is restricted in optAl cells. In optAl cells infected with phage $\mathrm{T} 7$ defective in gene $1.2, \mathrm{~T} 7 \mathrm{DNA}$ replication ceases prematurely and no viable phage are produced (6). Gene 1.2 encodes an inhibitor of dGTPase, enabling wild-type T7 phage to grow in $E$. coli optAl (7). The reduction in the dGTP pool can also explain the abortive infection of two mutants of bacteriophage T4, T4 dexA and T4 CB120 $(8,9)$, in E. coli optAl.

Biochemical characterization of the dGTPase has defined several interesting properties, but the physiological role of this enzyme is not known. For example, (i) dGTPase preferentially hydrolyzes dGTP over the other canonical NTPs (1-3); (ii) the association of dGTPase and its inhibitor, the gene 1.2 protein, is modulated by a complex mechanism (4, 7); (iii) dGTPase has a high affinity for DNA $(2,3)$; and (iv) the expression of the dGTPase gene, as evidenced by the optAl mutation, can vary 50 -fold. To further our understanding of the function of dGTPase in vivo, and to determine the basis of the increase in dGTPase in optAl cells, we have isolated and characterized the dGTPase gene* from both $E$. coli optA $A^{+}$and $E$. coli optAl. Quirk et al. (10) have localized the dGTPase gene from wild-type $E$. coli on a phage $\lambda$ clone

The publication costs of this article were defrayed in part by page charge payment. This article must therefore be hereby marked "advertisement" in accordance with 18 U.S.C. $\$ 1734$ solely to indicate this fact. containing an insert from the 3.6-min region of the $E$. coli chromosome.

\section{MATERIALS AND METHODS}

Bacterial Strains. E. coli HR42 (opt $\left.A^{+}\right)$and HR44 (optAl) are isogenic except for the optAl mutation (6). E. coli MV1190 (11) and E. coli HMS254 (hsdR thr- leu lac $^{-}$thi ${ }^{-}$ supE $E^{-}$dapD4 optAl tonA ${ }^{-}$) were from S. Tabor (Harvard Medical School). E. coli DH1 (12) was from M. Connor (University of California, Irvine). E. coli CG1090 (W3110 galE $^{-}$with a kanamycin-resistance minitransposon insertion $92 \%$ linked to optAl by $\mathrm{P} 1$ transduction) was from $\mathrm{C}$. Georgopoulus (University of Utah). E. coli HS40 was constructed by $\mathrm{H}$. Nakai (this laboratory) by transducing HR42 to kanamycin resistance with a lysate of P1vir grown on $E$. coli CG1090. E. coli MC48 (CP366 thr leu $^{-}$his $^{-}$rpsL $^{-}$lac $^{-}$ $\mathrm{xyl}^{-}$ara $^{-}$tonA $\mathrm{A}^{-}$tsx $\mathrm{thi}^{-} \mathrm{rha}^{-}$zig::Tn10 polAts 12) was from M. Carson (Harvard Medical School). E. coli SW8 and SW9 were constructed by transducing HR42 and HR44, respectively, to tetracycline resistance with a lysate of P1vir grown on $E$. coli MC48.

Bacteriophages and Plasmids. $\lambda$ EMBL4 clones (15A7, 9H2, 4E4, 23G6, 12D5) containing DNA inserts from the 3.6-min region of the $E$. coli chromosome were the gift of Yuji Kohara (Nagoya University). Plasmid pCD5, from C. Richaud (Institut de Microbiologie, Universite Paris-Sud), is a pBR322 plasmid with a 14-kilobase (kb) BamHI-HindIII fragment of $E$. coli chromosomal DNA that contains dapD.

DNA Methods. $E$. coli HR42 and HR44 chromosomal DNA was isolated as described (13) except that the $\mathrm{CsCl}$ step was omitted and the DNA was treated with RNase A at $50 \mu \mathrm{g} / \mathrm{ml}$ and then with proteinase $\mathrm{K}$ at $100 \mu \mathrm{g} / \mathrm{ml}$ in $0.5 \% \mathrm{NaDodSO}_{4}$. Phage $\lambda$ DNA iaolation (14) and Southern blot transfers (13) were carried out as described. Hybridizations with the degenerate 17-mer oligodeoxynucleotide ( $5^{\prime}$-TGCCARTTDATYTTYTT-3') were as described (14) with incubations at room temperature. Nitrocellulose filters were washed to a stringency of $0.3 \mathrm{M} \mathrm{NaCl} / 30 \mathrm{mM}$ sodium citrate with $0.05 \%$ sodium pyrophosphate. Radioactively labeled nucleotides were from New England Nuclear.

DNA Sequence Analysis. The nucleotide sequence was determined by the dideoxy chain-termination method as described in the Sequenase kit from United State Biochemical. The M13 universal primer (-20) was from New England Biolabs. Other primers were provided by A. Nussbaum (Harvard Medical School). pSW1 DNA was prepared (15) and used to determine the sequence of the $3^{\prime}$ end of the dGTPase gene on one strand. Double-stranded DNA was alkali-denatured. Template and primer were annealed in 75 $\mathrm{mM}$ Tris $\cdot \mathrm{HCl}$, $\mathrm{pH} 7.5 / 50 \mathrm{mM} \mathrm{MgCl}_{2}$ for $15 \mathrm{~min}$ at $37^{\circ} \mathrm{C}$.

Abbreviation: PCR, polymerase chain reaction.

*The sequence reported in this paper has been deposited in the GenBank data base (accession no. M31772). 
Polymerase Chain Reaction (PCR). Reactions were carried out according to Perkin-Elmer/Cetus with 3-50 $\mu$ g of DNA and Thermus aquaticus (Taq) DNA polymerase (PerkinElmer/Cetus). The dGTPase structural gene from $E$. coli HR44 (optA1) was amplified as a 1606-base-pair (bp) fragment by using primers derived from the sequence of the $E$. coli optA ${ }^{+}$dGTPase gene. The primers specific for the $5^{\prime}$ end (5'-CCGGATCCGGGGAAGCGTATTTCTCACGC-3') and for the $3^{\prime}$ end (5'-CCGAATTCCGAACTAAAGTTTCTGC$\left.3^{\prime}\right)$ each contained eight additional bases to include BamHI and EcoRI restriction sites (underlined), respectively. The amplified DNA was digested with BamHI and EcoRI. The 1600 -bp fragment was cloned into M13mp18 and M13mp19. To sequence the region upstream of the dGTPase gene in $E$. coli optA1, two primers (5'-TGTTCGGTTTTAACGCCCTG$3^{\prime}$ and 5'-CAGGCTGGACTGTTTAGCGG-3') were used to amplify an 819-base sequence. The double-stranded 819-bp fragment was isolated, digested with $T a q$ I to generate a 563-bp fragment, and cloned into M13mp19. For direct sequencing, single-stranded DNA of the same region was prepared by asymmetric PCR amplification (16) with the primers present in a ratio of 20:1 with respect to one another.

Enzymes. Most restriction enzymes were from New England Biolabs. T4 polynucleotide kinase and Sma I were from United States Biochemical. E. coli DNA polymerase I was from Pharmacia. Calf-intestine alkaline phosphatase was from Boehringer Mannheim.

Genetic Techniques. To replace the mutation in $E$. coli HR44 (optA1) with the analogous region from $E$. coli HR42 $\left(o p t A^{+}\right)$, an approach similar to that of Lee et al. (17) was used. A HindIII-EcoRI fragment containing the opt $A^{+} 1.5-\mathrm{kb}$ Stu I-Kpn I segment flanked by M13mp18 polylinker sequences was cloned into plasmid pBR322 to generate pSK. $E$. coli SW9 (polA ${ }^{\text {ts }}$ optAl) was transformed with $\mathrm{pSK}$ or pBR322 and transformants resistant to ampicillin $(25 \mu \mathrm{g} / \mathrm{ml})$ at $30^{\circ} \mathrm{C}$ were isolated. Transformants were plated at $42^{\circ} \mathrm{C}$ in the presence of ampicillin $(25 \mu \mathrm{g} / \mathrm{ml})$ to isolate plasmid integrates. pSK yielded ampicillin-resistant colonies at $42^{\circ} \mathrm{C}$ at a frequency of $1 / 2500$, whereas the frequency of integration of pBR322 was $<1 \times 10^{-6}$. To obtain ampicillinsensitive segregates, plasmid integrates were grown without ampicillin at $30^{\circ} \mathrm{C}$ and plated at $42^{\circ} \mathrm{C}$. Fourteen ampicillinsensitive colonies were identified among the 12,000 screened (Bio-Rad AmpScreen kit). The optAl phenotype was scored with wild-type phage T7 or T7 ST16 (deleted for gene 1.2).

Assay of dGTPase. Cells from $25 \mathrm{ml}$ of culture were resuspended in $0.8 \mathrm{ml}$ of cold $50 \mathrm{mM}$ Tris $\mathrm{HCl}, \mathrm{pH} \mathrm{7.5/10 \%}$ sucrose. Samples were treated with lysozyme $(1 \mathrm{mg} / \mathrm{ml})$ for $15 \mathrm{~min}$ on ice and centrifuged $(16,000 \times \mathrm{g})$ for $15 \mathrm{~min}$ at $4^{\circ} \mathrm{C}$. The supernatant was assayed for dGTPase activity as described (3).

\section{RESULTS}

Identification and Cloning of the dGTPase Structural Gene. Our approach to identify the dGTPase gene was based on the assumption that it contained or lay in close proximity to the optAl mutation. Since the concentration of dGTPase protein is increased 50 -fold as a consequence of the optAl mutation $(3,4)$, we considered it likely that the mutation affected a regulatory region, leading to increased transcription of the dGTPase gene. Since optAl maps to the 3.6-min region on the $E$. coli linkage map (6), the dGTPase gene should be located near dapD; dapD is $92 \%$ cotransducible with optAl (6). On this assumption, a series of $\lambda$ clones containing inserts of DNA from the 3.6-min region of the $E$. coli chromosome (18) were screened for the presence of the dGTPase gene. pCD5, a pBR322 plasmid with a 14-kb BamHI-HindIII insert containing dapD, was included in this analysis. The $\mathrm{N}$-terminal amino acid sequence of the dGTPase protein $(2,3)$ was used

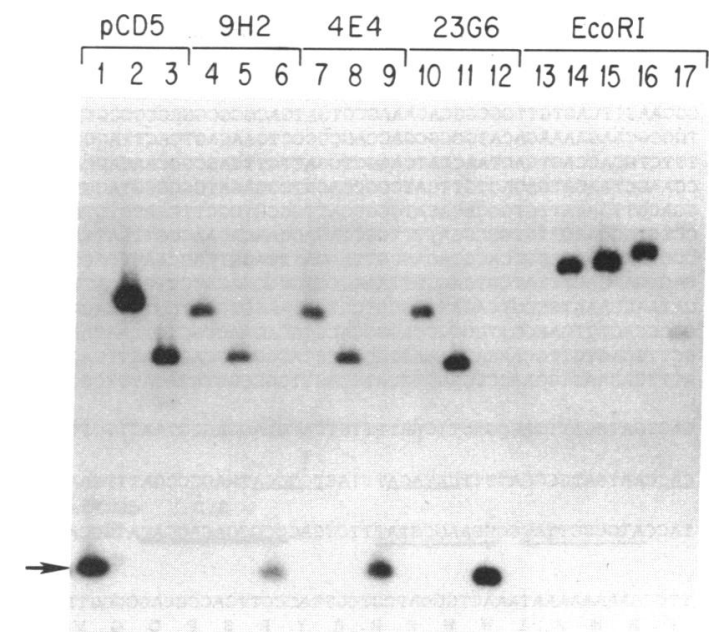

FIG. 1. Southern blot analysis of selected $\lambda$ EMBL4 clones (15A7, 9H2, 4E4, 23G6, 12D5) and the plasmid pCD5 cleaved with various restriction enzymes and hybridized to a degenerate 17-mer. Lanes 1, 6, 9, and 12, Pst I and Pvu II; lanes 2, 4, 7, and 10, Pst I; lanes 3, 5, 8, and 11, Pvu II; lanes 13-17, EcoRI. The DNAs used in lanes 13-17 were $\lambda$ clones 15A7 (lane 13), 9H2 (lane 14), 4E4 (lane 15), 23G6 (lane 16), and 12D5 (lane 17). Arrow indicates the 1.5-kb $P v u$ II-Pst I fragment that contains the N terminus of the dGTPase structural gene.

to synthesize a degenerate 17-base oligonucleotide, which, in turn, was used in Southern blots to probe restriction digests of five $\lambda$ clones and pCD5. Three of the $\lambda$ clones (9H2, 23G6, and 4E4) and pCD5 hybridized to the probe (Fig. 1). Hybridization to pCD5 localized the dGTPase gene to within $8 \mathrm{~kb}$ of dapD.

In order to clone the dGTPase gene and possibly the optAI mutation from the isogenic strains, $E$. coli HR42 (opt $\left.A^{+}\right)$and HR44 (optA1), we compared restriction digest maps of the 3.6-min region of the $E$. coli chromosome provided by $Y$. Kohara (18) and pCD5 from C. Richaud (personal communication). This analysis indicated that a 15-kb HindIII fragment contained both the dGTPase structural gene and dapD. $H$ indIII fragments in this size range were isolated from $E$. coli HR42 and HR44 chromosomal digests and cloned into
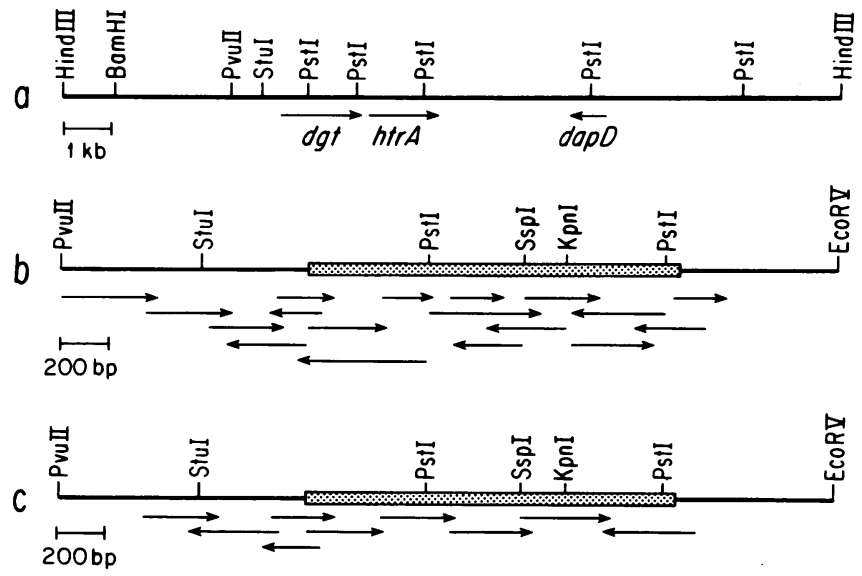

FIG. 2. (a) Restriction digest map of the $E$. coli HR42 15.8-kb HindIII fragment isolated from the dapD ampicillin-resistant transformant pSW1. (b) Sequencing strategy of the wild-type dGTPase gene and flanking sequences. The fragments from pSW1 used for sequencing the dGTPase gene were $1.5 \mathrm{~kb} P v u$ II-Pst I, $0.98 \mathrm{~kb} P s t$ I, 1.5 kb Stu I-Kpn I, and 0.7 kb Pst I-EcoRV. (c) Sequencing strategy of the dGTPase gene from E. coli HR44 (optAl). 
PvuII

1 CAGCTGGTCAACCTGCCGCTCGGCATTACCTCCACGCCAGGCAATCACCAGATCGGGTTT 61 CAGCGCGACAATGCGTTCCAGATTCATCCCCTGCCAGGCGAAACCTGCTCAATCTTTTG 121 CGCTTGTGGAGGATAGTCGGAATAGCTGCTGACCCCAACCGGCGTGATCCCGGCGGCAAA 181 GGCAAGTTCAGTGTTGGCGGGAGAAGCGTGATGACGCGCGGCGCGGCGTTGAGCCACAG 241 TGGCGCAAGAAAAGACATGGGGCGACCAGCGCCCTGAACAGTGACTTAGCCATGTGCAAG 241 TGGCGCAAGAAAAGACATGGGGCGACCAGCGCCCTGAACAGTGACT TAGCCATGTGCAAG 301 TTTCTGCACCAGTGACTAACCATCAGGCTGGACTGTTTAGCGGCAACAGCAGGAACTCAT 421 GGACGTTGAAATTGTGGCAGACATGGGCGATTGCCGTCGCTTCCATCTCTACAGCAATGG 481 CCTGTGGGAAGTTGTGGCGGATTTTCGCCAGACCAACAGAACCGTTGATGAAAGCGTCGC 541 CGCTAACAATCAGGCCACGTACAGCGTTAAGATTCAGTTCGGCAATGCAGGCCTCAGCGG 601 CAGCGATCAGTTTATCGTCAGCTTTAAAGCCTGCCGGACACCCTGGTAACTGACCGTATT 721 CGCCCACTTTCAACGTTGGTGCCAGGCCACCGGCAGAACCGGTGTTAATAATCACATCTG 781 GCTTGCAGTGTTCCAACAGCAAAGTGGCACCCAGCGCCGCAGCGACTTTACCGATGCCCG 841 ATTTCAGAAGCGCAACCTCGGTTCCATTCAGTTGGCCGGTATAGATTTCGCAACCGCCGA

901 GACTGATAGTTTGACGGTTTTCGATTTTGTCACGCAGCAGCGTAACTTCTTCTTCCA ATTE

961 CACCAATGATGCCGATTTTCATAGATTTACTC்GCGATAAGCCCGATTTGAAGGCATAGTT S.D. dGTPase

1021 TACCATGCGCTTACGGGGAGCGTATTTCTCACGCGGGAGAGGACATGGCACAGATTGAT $M \quad A \quad I \quad D$

1081 TTCCGAAAAAAAATAAACTGGCATCGTCGTTACCGTTCACCGCAGGGCGTTAAAACCGAA $\begin{array}{lllllllllllllllllllll} & F & R & K & K & I & N & W & H & R & R & Y & R & S & P & Q & G & V & K & T & E\end{array}$

1141 CATGAGATCCTGCGGATCTTCGAGAGCGATCGCGGGCGTATCATCAACTCTCCGGCAATT $\begin{array}{lllllllllllllllllllll}H & E & I & \text { L } & R & \text { I } & F & \text { E } & S & D & R & G & R & I & I & N & S & P & A & I\end{array}$

1201 CGTCGTCTGCAACAAAAGACCCAGGTTTTTCCACTGGAGCGCAATGCCGCCGTGCGCACG $\begin{array}{llllllllllllllllllll}R & R & L & Q & Q & K & T & Q & V & F & P & \text { L } & \text { E } & R & \text { N } & A & A & V & R & T\end{array}$

1261 CGTCTTACCCACTCGATGGAAGTCCAGCAGGTGGGGCGCTACATCGCCAAAGAAATTTTA $\begin{array}{llllllllllllllllllll}R & \text { I } & T & H & S & M & E & V & 0 & 0 & V & G & R & Y & I & A & K & E & I & L\end{array}$

1321 AGCCGTCTGAAAGAGCTTAAATTACTGGAAGCATACGGCCTGGATGAACTGACCGGTCCC $\begin{array}{llllllllllllllllllll}S & R & L & K & E & L & K & L & L & E & A & Y & G & L & D & E & L & T & G & P\end{array}$

1381 TTTGAAAGCATTGTTGAGATGTCATGCCTGATGCACGATATCGGCAATCCGCCGTTTGGT

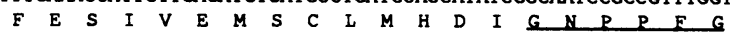

1441 CATTTTGGCGAAGCGGCGATAAATGACTGGTTTCGCCAACGTTTGCACCCGGAAGATGCC $\begin{array}{llllllllllllllllllll}H & F & G & E & A & A & I & N & D & W & F & R & Q & R & \text { L } & H & P & E & D & A\end{array}$

1501 GAAAGCCAGCCTCTGACTGACGATCGCTGCAGCGTGGCGGCACTACGTTTACGGGACGGG

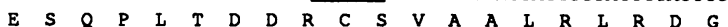

1561 GAAGAACCGCTTAACGAGCTGCGGCGCAAGATTCGTCAGGACTTATGTCATTTTGAGGGG $\begin{array}{llllllllllllllllllll}E & E & P & L & N & E & L & R & R & K & I & R & Q & D & L & C & H & F & E & G\end{array}$

1621 AATGCACAAGGCATTCGCCTGGTGCATACATTGATGCGGATGAATCTCACCTGGGCACAG

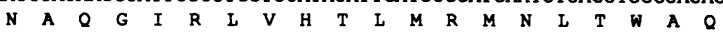

1681 GTTGGCGGTATTTTAAAATATACCCGTCCGGCGTGGTGGCGTGGCGAAACGCCTGAGACA $\begin{array}{llllllllllllllllllllll}V & G & G & I & L & K & Y & T & R & P & A & W & W & R & G & E & T & P & E & T\end{array}$

1741 CATCACTATTTAATGAAAAAGCCGGGTATTATCTTTCTGAAGAAGCCTATATTGCCCGG $\begin{array}{llllllllllllllllllllll}\text { H } & \text { H } & \text { Y } & \text { L } & \text { M } & \text { K } & \text { K } & \text { P } & \text { G } & \text { Y } & \text { Y } & \text { L } & \text { S } & \text { E } & \text { E } & \text { A } & \text { Y } & \text { I } & \text { A } & R\end{array}$

1801 TTGCGTAAAGAACTTAATTTGGCGCTTTACAGTCGTTTTCCATTAACGTGGATTATGGAA $\begin{array}{lllllllllllllllllllllll}L & R & K & E & \text { L } & N & \text { L } & A & \text { L } & \text { Y } & S & \text { S } & \text { F } & \text { P } & \text { L } & \text { T } & \text { W } & \text { I } & M & \text { M }\end{array}$

1861 GCTGCCGACGACATCTCCTATTGTGTGGCAGACCTTGAAGATGCGGTAGAGAAAAGAATA $\begin{array}{llllllllllllllllllllll}A & A & D & D & I & S & Y & C & V & A & D & \text { L } & E & D & A & V & E & K & R & I\end{array}$

1921 TTTACCGTTGAGCAGCTTTATCATCATTTGCACGAAGCGTGGGGCCAGCATGAGAAAGGT

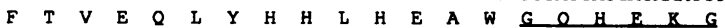

1981 TCGCTCTTTTCGCTGGTGGTTGAAAATGCCTGGGAAAAATCACGCTCAAATAGTTTAAGC $\begin{array}{llllllllllllllllllll}S & L & F & S & L & V & V & E & N & A & W & E & K & S & R & S & N & S & L & S\end{array}$

2041 CGCAGTACGGAAGATCAGTTTTTTATGTATTTACGGGTAAACACCCTAAATAAACTGGTA $\begin{array}{llllllllllllllllllll}R & S & T & E & D & Q & F & F & M & Y & L & R & V & N & T & L & N & K & L & V\end{array}$

2101 CCCTACGCGGCACAACGATTTATTGATAATCTGCCTGCGATTTTCGCCGGAACGTTTAAT $\begin{array}{llllllllllllllllllll}P & Y & A & A & Q & R & F & I & D & N & \text { L } & P & A & I & F & A & G & T & F & N\end{array}$

2161 CATGCATTATTGGAAGATGCCAGCGAATGCAGCGATCTTCTTAAGCTATATAAAAATGTC $\begin{array}{lllllllllllllllllllll}\text { H } & \text { A } & \text { L } & \text { L } & \text { E } & \text { D } & \text { A } & \text { S } & \text { E } & \text { C } & \text { S } & \text { D } & \text { L } & \text { L } & \text { K } & \text { L } & \text { Y } & \text { K } & \text { N } & \text { V }\end{array}$

2221 GCTGTAAAACATGTGTTTAGCCATCCAGATGTCGAGCGGCTTGAATTGCAGGGCTATCGG $\begin{array}{llllllllllllllllllll}A & V & K & H & V & F & S & H & P & D & V & E & R & I & E & L & O & G & Y & R\end{array}$

2281 GTCATTAGCGGATTATTAGAGATTTATCGTCCTTTATTAAGCCTGTCGTTATCAGACTTT $V$ I S G I I E I Y R P I I S I S L S D F

2341 ACTGAACTGGTAGAAAAAGAACGGGTGAAACGTTTCCCTATTGAATCGCGCTTATTCCAC $\begin{array}{lllllllllllllllllllll}T & E & L & V & E & K & E & R & V & K & R & F & P & I & E & S & R & L & F & H\end{array}$

2401 AAACTCTCGACGCGCCATCGGCTGGCCTATGTCGAGGCTGTCAGTAAATTACCGTCAGAT $\begin{array}{llllllllllllllllllllll}K & \text { L } & \text { S } & \text { T } & \text { R } & \text { H } & \text { R } & \text { L } & \text { A } & \text { Y } & \text { V } & \text { E } & \text { A } & \text { V } & \text { S } & \text { K } & \text { L } & \text { P } & \text { S } & \text { D }\end{array}$

2461 TCTCCTGAGTTTCCGCTATGGGAATATTATTACCGTTGCCGCCTGCTGCAGGATTATATC $\begin{array}{llllllllllllllllllll}S & P & E & F & P & L & W & E & Y & Y & Y & R & C & R & L & L & O & D & Y & I\end{array}$

2521 AGCGGTATGACCGACCTCTATGCGTGGGATGAATACCGACGTCTGATGGCCGTAGAACAA $\begin{array}{llllllllllllllllllll}S & G & M & T & D & L & Y & A & W & D & E & Y & R & R & L & M & A & V & E & 0\end{array}$

2581 TAACCAGGCTTTTGTAAAGACGAACAATAAATTTTTACCTTTTGCAGAAACTTTAGTTCG

2641 GAACTTCAGGCTATAAAACGAATCTGAAGAACACAGCAATTTTGCGTTATCTGTTAATCG htrA

2701 AGACTGAAATACATGAAAAAAACCACATTAGCACTGAGTCGACTGGCTCTGAGTTTAGGT $\begin{array}{llllllllllllllll}M & K & K & T & T & L & A & \text { L } & S & R & \text { L } & \text { A } & \text { L } & \text { S } & \text { L } & G\end{array}$
pBR322. Recombinant plasmids containing dapD were isolated by transforming E. coli HMS254 (optAl dapD4) and selecting for dapD ampicillin-resistant transformants. dapD4 mutants normally require exogenous diaminopimelic acid for growth (19). Six dapD ampicillin-resistant transformants were obtained from clones with HR42 DNA but none with HR44 DNA. Restriction digest and Southern blot analysis of the dapD ampicillin-resistant transformants with HR42 inserts showed that all six contained the same HindIII insert and the dGTPase structural gene (data not shown). One of these clones, $\mathrm{pSW} 1$, was used in all subsequent experiments. Using a similar approach, Quirk et al. (10) also identified a $\lambda$ clone with an insert carrying the dGTPase gene. We have maintained the mnemonic dgt for the dGTPase structural gene.

An extract prepared from DH1 cells harboring pSW1 was analyzed by Western blot using polyclonal antibodies to purified dGTPase. These extracts contain a 5- to 10-fold higher level of a 59-kDa protein that comigrates with dGTPase from E. coli HR44 extracts in denaturing polyacrylamide gels.

When $\mathrm{pSW} 1$ is introduced into $E$. coli HR42, the level of dGTPase activity in extracts increases 5-fold. These cells, however, are not optAl as judged by their ability to support the growth of $T 7$ gene 1.2 mutant phage. Quirk et al. (10), however, demonstrated that $E$. coli strains harboring a plasmid similar to pSW1 did not support the growth of phage T4 $d s d$ mutants (8), indicating that the strain had become phenotypically optA1. We believe that this difference stems from a difference in plasmid copy number and/or from the difference in phage used to screen for the optAl phenotype. Surprisingly, we observed that $E$. coli HR42 is phenotypically optAl when the 4.1-kb HindIII-Stu I fragment (see Fig. 2a) is deleted from pSW1 (data not shown).

DNA Sequence Analysis of the dGTPase Gene. Southern blot analysis of restriction digests of pCD5 showed that the sequence encoding the $\mathrm{N}$ terminus of the dGTPase resided on a 1.5-kb Pvu II-Pst I fragment (Fig. 1). The corresponding fragment was isolated from the HR42 insert in pSW1 to carry out DNA sequence analysis as outlined in Fig. $2 b$.

The nucleotide sequence of the dGTPase structural gene and the upstream region is shown in Fig. 3. The open reading frame codes for a 505-amino acid protein whose deduced amino acid sequence is in agreement with the $\mathrm{N}$-terminal sequence determined from the purified protein $(2,3)$. The calculated molecular weight of the dGTPase is 59,315 , similar to that determined for the purified protein $(2,3)$. Searches of the Protein Identification Resource data bank of the National Biomedical Resource Foundation (June 1989) and the GenBank data base (release 61.0) did not identify any proteins or nucleotide sequences with significant homology to dGTPase.

FIG. 3. Nucleotide sequence and potential regulatory elements of the dGTPase gene $(d g t)$. The nucleotide sequence shown begins at the $P v u$ II site 1066 nucleotides upstream of the dGTPase translation initiation site. The Shine-Dalgarno ribosome binding site (S.D.) is underscored with a heavy line. The region of dyad symmetry in the presumptive promoter region is indicated by the inverted arrows. The $C \rightarrow T$ transition identified in $E$. coli HR44 is indicated 74 nucleotides upstream of the dGTPase translation initiation codon. The -35 and -10 regions of the potential $E$. coli RNA polymerase promoters are underlined. The two Gly-Xaa-Xaa-Xaa-Xaa-Gly loops are underlined below the predicted amino acid sequence of dGTPase. Downstream of the dGTPase termination codon, the A+T-rich region is indicated by arrows, followed by $h \operatorname{tr} A$; the $h \operatorname{trA} \sigma \mathrm{E}$ promoter is underlined and the beginning of the htrA translation product is shown (20). Upstream of the dGTPase regulatory region, ORF indicates the initiation codon of the potential gene that is oriented in the direction opposite to that of $\mathbf{d g t}$. 
Immediately upstream of the translation initiation site is a Shine-Dalgarno ribosome binding site (21). Also present are two regions with homology to the consensus recognition sequence for the $E$. coli RNA polymerase holoenzyme, which consists of TTGACA as the -35 sequence and TATAAT as the -10 sequence $(22)$. The region $\approx 50 \mathrm{bp}$ upstream of the $d g t$ translational initiation site ( -35 TCGCGA, -10 CATAGT) has stronger homology than the region 80 bp upstream $(-35$ TTGCAC, -10 CATAGA). A short region of dyad symmetry lies between the -10 CATAGT sequence and the translational start codon. Immediately following the termination codon of dgt is an A+T-rich sequence containing dyad symmetry. One hundred and thirty base pairs downstream of $d g t$ is $h t r A(\operatorname{degP})$, a gene encoding a periplasmic protease required for growth of $E$. coli at elevated temperatures (20, 23). htrA has a potential $\sigma \mathrm{E}$ promoter (20) and does not appear to form an operon with $d g t$.

A second, nonoverlapping open reading frame with the coding potential for a protein of $24 \mathrm{kDa}$ exists upstream of $d g t$ (see Fig. 3, ORF). If expressed, this gene would be transcribed in the direction opposite to that of $d g t$ transcription, and its promoter region would overlap that of $d g t$.

Characterization of the optAI Mutation. Attempts to clone the region analogous to pSW1 from $E$. coli optAl into a multicopy vector have been unsuccessful. We therefore took advantage of the known sequence of this region from wildtype $E$. coli to use PCR to determine the nucleotide sequence of the dGTPase gene and the upstream region in E. coli optAl DNA. The sequence of $d g t$ from optAl cells is identical to that of the wild-type dGTPase gene. Sequencing of the presumptive promoter region revealed a single nucleotide change: a $\mathrm{C} \rightarrow \mathrm{T}$ transition 74 nucleotides upstream of the dGTPase translation initiation site (see Fig. 3). This $\mathrm{C} \rightarrow \mathrm{T}$ transition removes an $N r u$ I site, providing a convenient screen for its presence.

The Promoter Mutation Is Necessary for Expression of the optA1 Phenotype. In order to determine whether the $\mathrm{C} \rightarrow \mathrm{T}$ transition identified in $E$. coli HR44 was necessary for expression of the optAl phenotype, the region containing this mutation in $E$. coli HR44 was replaced by homologous recombination with that from $E$. coli $\mathrm{HR} 42\left(o p t A^{+}\right)$. pSK is a pBR322 plasmid with a 1.5-kb Stu I-Kpn I fragment (see Fig. 2a) from $E$. coli HR42. The only difference between the cloned sequence in pSK and the chromosomal sequence in $E$. coli HR44 is the $\mathrm{C} \rightarrow \mathrm{T}$ transition in a potential promoter region of $d g t$.

Fourteen colonies that had integrated and then resolved pSK from the chromosome were analyzed for their optAl phenotype. The region encompassing the mutation was PCRamplified from chromosomal DNA to yield an 819-bp fragment, which was then digested with Nru I to test for the presence of the optAl mutation. Six of the 14 colonies had lost the mutation. These same 6 colonies also recovered the ability to support the growth of T7 phage deficient in gene 1.2. Extracts prepared from 2 of the 6 colonies had levels of dGTPase activity comparable to that in $E$. coli SW8 (opt $\left.A^{+}\right)$ (Table 1). Western blot analysis of extracts prepared from

Table 1. dGTPase activity in extracts of $E$. coli. cells

\begin{tabular}{|c|c|}
\hline Strain & $\begin{array}{l}\text { Activity, pmol of dGTP } \\
\text { hydrolyzed per } 30 \mathrm{~min} \\
\text { per mg of protein }\end{array}$ \\
\hline SW8 $\left(o p t A^{+}\right)$ & 4.7 \\
\hline SW9 (optAl) & 120 \\
\hline SW9 optA ${ }^{+}$(isolate 1 ) & 5.2 \\
\hline SW9 optA ${ }^{+}$(isolate 2) & 4.8 \\
\hline HS40 (dgt::mini-Tn10) & 2.1 \\
\hline
\end{tabular}

Extracts (0.3-8.4 $\mu \mathrm{g}$ of protein) were tested for their ability to catalyze the hydrolysis of ${ }^{32} \mathrm{P}$ from $\left[\alpha-{ }^{32} \mathrm{P}\right] \mathrm{dGTP}(3)$.

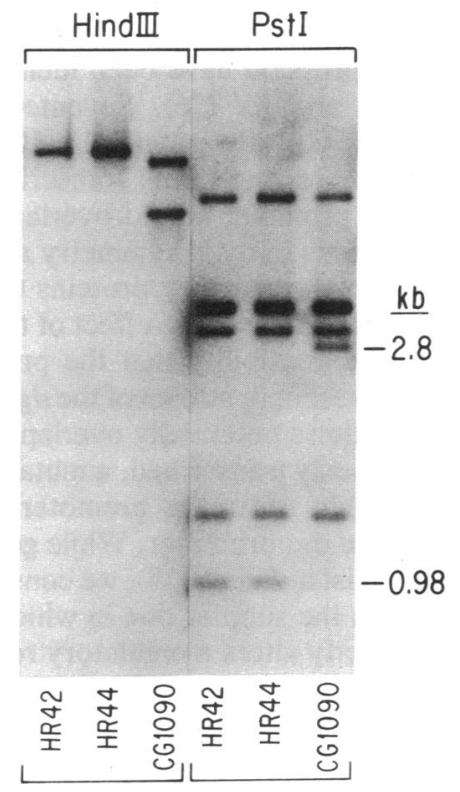

Fig. 4. Southern blot analysis to localize the kanamycinresistance minitransposon insertion linked to htrA and optAl in $E$. coli CG1090. Bacterial chromosomal DNA was digested with HindIII or Pst I and probed with nick-translated pSW1. Lanes 1 and 4, E. coli HR42; lanes 2 and 5, E. coli HR44; lanes 3 and 6, E. coli CG1090. The HindIII digest of CG1090 contains an additional fragment due to the presence of a HindIII site within the minitransposon insertion (24). In the Pst I digest of CG1090, the 0.98-kb Pst I fragment containing the majority of the dGTPase coding region is replaced by a $2.8-\mathrm{kb}$ fragment due to the minitransposon insertion.

these two strains confirmed that they no longer overproduced dGTPase (data not shown). We conclude that the $\mathrm{C} \rightarrow \mathrm{T}$ transition present in $E$. coli HR44 is required for expression of the optAl phenotype.

The dGTPase Gene Is Not Essential. We have characterized a second $E$. coli dGTPase mutant. $E$. coli $\mathrm{CG} 1090$ and $E$. coli HS40 both contain a 1.8-kb kanamycin-resistance minitransposon insertion that disrupts the dGTPase coding (Fig. 4). No dGTPase mRNA transcripts were detected in a Northern blot analysis of RNA from $E$. coli CG1090 (data not shown). The level of dGTPase activity in extracts of $E$. coli HS40 (dgt::mini-Tn10) is less than half that in extracts of $E$. coli SW8 $\left(o p t A^{+}\right)$(Table 1). Presumably, the residual dGTPase activity present in $E$. coli HS40 extracts is due to phosphatases with other specificities. Thus, $E$. coli CG1090 and $E$. coli $\mathrm{HS} 40$ are null mutants of dGTPase. Since these strains have no detectable phenotype in LB medium (14) at $30-42^{\circ} \mathrm{C}$, an intact $d g t$ gene is not required for bacterial growth under these conditions.

\section{DISCUSSION}

We have characterized the gene encoding dGTPase from $E$. coli HR42 (wild-type) and from $E$. coli HR44 (optA1), a strain that overexpresses dGTPase. Sequence analysis of the dGTPase gene (dgt) from optAl has ruled out the possibility that a mutation in the structural gene gives rise to the 50-fold higher levels of dGTPase activity. DNA sequence analysis of the $d g t$ region from $E$. coli optAl identified only one nucleotide change, a $\mathrm{C} \rightarrow \mathrm{T}$ transition 74 nucleotides upstream of the dGTPase translation initiation site (Fig. 3). This mutation, which is required for overexpression of the dGTPase, is in a position to alter a potential transcriptional promoter of $d g t$. Mutating TCGCGA to TTGCGA increases the homology of this sequence to the -35 consensus recognition sequence, TTGACA, of the $E$. coli $\sigma^{70}$ RNA polymerase holoenzyme 
(22). Analogous mutations in the -35 region that result in large increases in expression have been identified in the $E$. coli genes lacI (25) and fol (26). Seventeen nucleotides downstream of TCGCGA is a potential -10 region of the promoter (Fig. 3). Interestingly, the transcription initiation site of this potential promoter would overlap the region of dyad symmetry. Regions of dyad symmetry are often recognition sites for bacterial regulatory proteins (27).

An alternative explanation of the effect of the mutation on dgt expression is the possibility that the promoter for the open reading frame present upstream of the $d g t$ gene overlaps the $d g t$ promoter. If these potentially overlapping promoters cannot be simultaneously transcribed, a mutation preventing transcription from this upstream promoter would permit transcription from the dgt promoter. While genes with overlapping promoters exist in $E$. coli (28), we consider this model to be less likely than the simpler one in which the mutation in optAl strains directly alters a regulatory region of the $d g t$ gene.

Although we have shown that the $\mathrm{C} \rightarrow \mathrm{T}$ transition is essential for the optAl phenotype, we do not know if it is sufficient. Since the original optAl strain (HR44) was isolated through nitrosoguanidine mutagenesis, it is possible that more than one mutation is required for expression of the optAl phenotype. Attempts to demonstrate that this single mutation is sufficient for expression of the optAl phenotype have thus far been inconclusive. The inability to clone the dGTPase gene from the optAl strain onto a multicopy vector also indicates that the mutation may be lethal under certain conditions.

What is the role of dGTPase? Under the conditions tested, dGTPase is not essential for the growth of $E$. coli; strain CG1090, containing a minitransposon insertion that disrupts the coding region of $d g t$, has no detectable phenotype. It is, of course, possible that $E$. coli $\mathrm{CG} 1090$ contains a partially active truncated dGTPase or that $E$. coli contains one or more additional proteins with comparable function.

Among possible nonessential roles for the dGTPase, the regulation of intracellular dGTP pools would appear to be an energetically wasteful pathway. It seems more likely that, in vivo, dGTPase may be part of a larger enzyme complex. For example, dGTPase activity may be coupled to other enzymatic activities such that the products of dGTP hydrolysis are utilized in other metabolic pathways. Alternatively, dGTP may not be the preferred substrate for the enzyme. Seto et al. (2) have proposed a preferential hydrolysis of dGTP in the syn configuration, a reaction that would decrease the chance of an $\mathbf{A} \cdot \mathbf{G}$ mispair during DNA replication. Related to these points is the question of whether the multiple conformational states observed during the interaction with $\mathrm{T} 7$ gene 1.2 protein are fortuitous or represent a need for control of dGTPase activity $(4,7)$.

Unfortunately, the amino acid sequence of the dGTPase offers little insight into these potential roles. dGTPase has two Gly-Xaa-Xaa-Xaa-Xaa-Gly loops (Fig. 3), a motif found in other nucleotide-binding proteins (29). A computer generated Chou-Fasman (30) analysis predicted that the first of these loops is more likely to lie within a region of secondary structure that resembles mononucleotide-binding folds (29). However, since previous experiments indicate that there is one NTP binding site for every two dGTPase monomers (4), it is possible that the mononucleotide-binding domain-is composed of residues from more than one monomer.

We gratefully acknowledge Stanley Tabor and Hiroshi Nakai for help with all aspects of this project. We thank John Rush for computer assistance and Hans Huber, Stanley Tabor, and Lynn Mendelman for critical reading of the manuscript. We thank $\mathrm{C}$. Georgopoulus for communicating results prior to publication and for bacterial strains. This work was supported by a Pharmacology Training Grant to S.M.W. and by grants from the American Cancer Society (NP-1P) and the U.S. Public Health Service (GAI06045).

1. Kornberg, S. R., Lehman, I. R., Bessman, M. J., Simms, E. S. \& Kornberg, A. (1958) J. Biol. Chem. 233, 159-162.

2. Seto, D., Bhatnagar, S. K. \& Bessman, M. J. (1988) J. Biol. Chem. 263, 1494-1499.

3. Beauchamp, B. B. \& Richardson, C. C. (1988) Proc. Natl. Acad. Sci. USA 85, 2563-2567.

4. Nakai, H. \& Richardson, C. C. (1990) J. Biol. Chem. 265, 4411-4419.

5. Myers, J. A., Beauchamp, B. B. \& Richardson, C. C. (1987) J. Biol. Chem. 262, 5288-5292.

6. Saito, H. \& Richardson, C. C. (1981) J. Virol. 37, 343-351.

7. Huber, H. E., Beauchamp, B. B. \& Richardson, C. C. (1988) J. Biol. Chem. 263, 13549-13556.

8. Gauss, P., Doherty, D. H. \& Gold, L. (1983) Proc. Natl. Acad. Sci. USA 80, 1669-1673.

9. Gauss, P., Gayle, M., Winter, R. \& Gold, L. (1987) Mol. Gen. Genet. 206, 24-34.

10. Quirk, S., Seto, D., Bhatnagar, S. K., Gauss, P., Gold, L. \& Bessman, M. J. (1989) Mol. Microbiol. 3, 1391-1395.

11. Tabor, S. \& Richardson, C. C. (1989) J. Biol. Chem. 264, 6447-6458.

12. Hanahan, D. (1983) J. Mol. Biol. 166, 557-580.

13. Ausubel, F. A., Brent, R., Kingston, R. E., Moore, D. D. Seidman, J. G., Smith, J. A. \& Struhl, K. (1987) Current Protocols in Molecular Biology (Wiley, New York)

14. Maniatis, T., Fritsch, E. F. \& Sambrook, J. (1982) Molecular Cloning: A Laboratory Manual (Cold Spring Harbor Lab., Cold Spring Harbor, NY).

15. Vogelstein, B. \& Gillespie, D. (1979) Proc. Natl. Acad. Sci. USA 76, 615-619.

16. Innis, M. A., Myambo, K. B., Gelfand, D. H. \& Brow, M. A. D. (1988) Proc. Natl. Acad. Sci. USA 85, 9436-9440.

17. Lee, C. A., Fournier, M. J. \& Beckwith, J. (1985) J. Bacteriol. 161, 1156-1161.

18. Kohara, Y., Akiyama, K. \& Isono, K. (1987) Cell 50, 495-508.

19. Bukhari, A. I. \& Taylor, A. L. (1971) J. Bacteriol. 105, 844854.

20. Lipinska, B., Sharma, S. \& Georgopoulus, C. (1988) Nucleic Acids Res. 16, 10053-10067.

21. Shine, J. \& Dalgarno, L. (1974) Proc. Natl. Acad. Sci. USA 71, 1342-1346.

22. Hawley, D. K. \& McClure, W. R. (1983) Nucleic Acids Res. $11,2237-2255$.

23. Strauch, K. L., Johnson, K. \& Beckwith, J. (1989) J. Bacteriol. 171, 2689-2696.

24. Way, J. C., Davis, M. A., Morisato, D., Roberts, D. E. \& Kleckner, N. (1984) Gene 32, 369-379.

25. Calos, M. P. (1978) Nature (London) 274, 762-765.

26. Smith, D. R., Rood, J. I., Bird, P. I., Sneddon, M. K., Calvo, J. M. \& Morrison, J. F. (1982) J. Biol. Chem. 257, 9043-9048.

27. Gunsalus, R. P. \& Yanofsky, C. (1980) Proc. Natl. Acad. Sci. USA 77, 7117-7121.

28. Pettis, G. S., Brickman, T. J. \& McIntosh, M. A. (1988) J. Biol. Chem. 263, 18857-18863.

29. Bradley, M. K., Smith, T. F., Lathrop, R. H., Livingston, D. M. \& Webster, T. A. (1987) Proc. Natl. Acad. Sci. USA 84, 4026-4030.

30. Chou, P. Y. \& Fasman, G. D. (1974) Biochemistry 13, 222-245. 